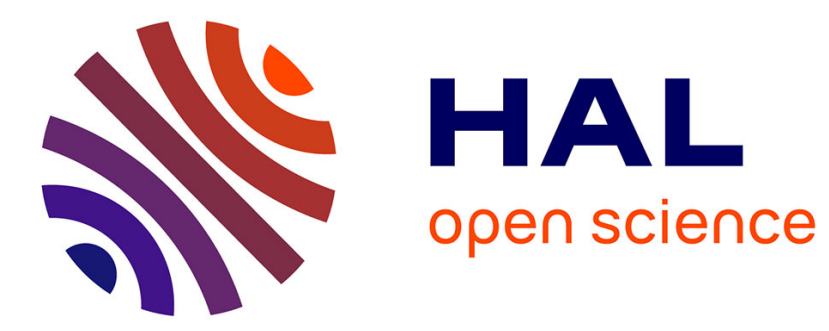

\title{
Producer's spatial equilibrium with a fuzzy constraint
}

Claude Ponsard

\section{To cite this version:}

Claude Ponsard. Producer's spatial equilibrium with a fuzzy constraint. [Research Report] Institut de mathématiques économiques (IME). 1980, 28 p., bibliographie. hal-01542346

\section{HAL Id: hal-01542346 \\ https://hal.science/hal-01542346}

Submitted on 19 Jun 2017

HAL is a multi-disciplinary open access archive for the deposit and dissemination of scientific research documents, whether they are published or not. The documents may come from teaching and research institutions in France or abroad, or from public or private research centers.
L'archive ouverte pluridisciplinaire HAL, est destinée au dépôt et à la diffusion de documents scientifiques de niveau recherche, publiés ou non, émanant des établissements d'enseignement et de recherche français ou étrangers, des laboratoires publics ou privés. 


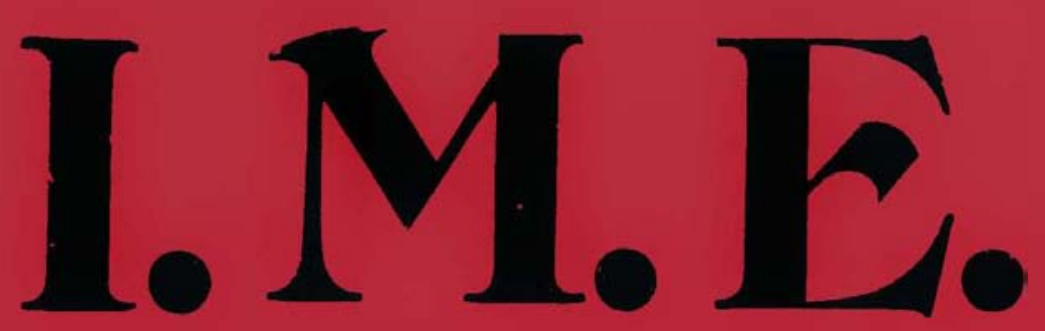

EQUIPE DE RECHERCHE ASSOCIEE AU C.N.R.S.

DOCUMENT DE TRAVAIL

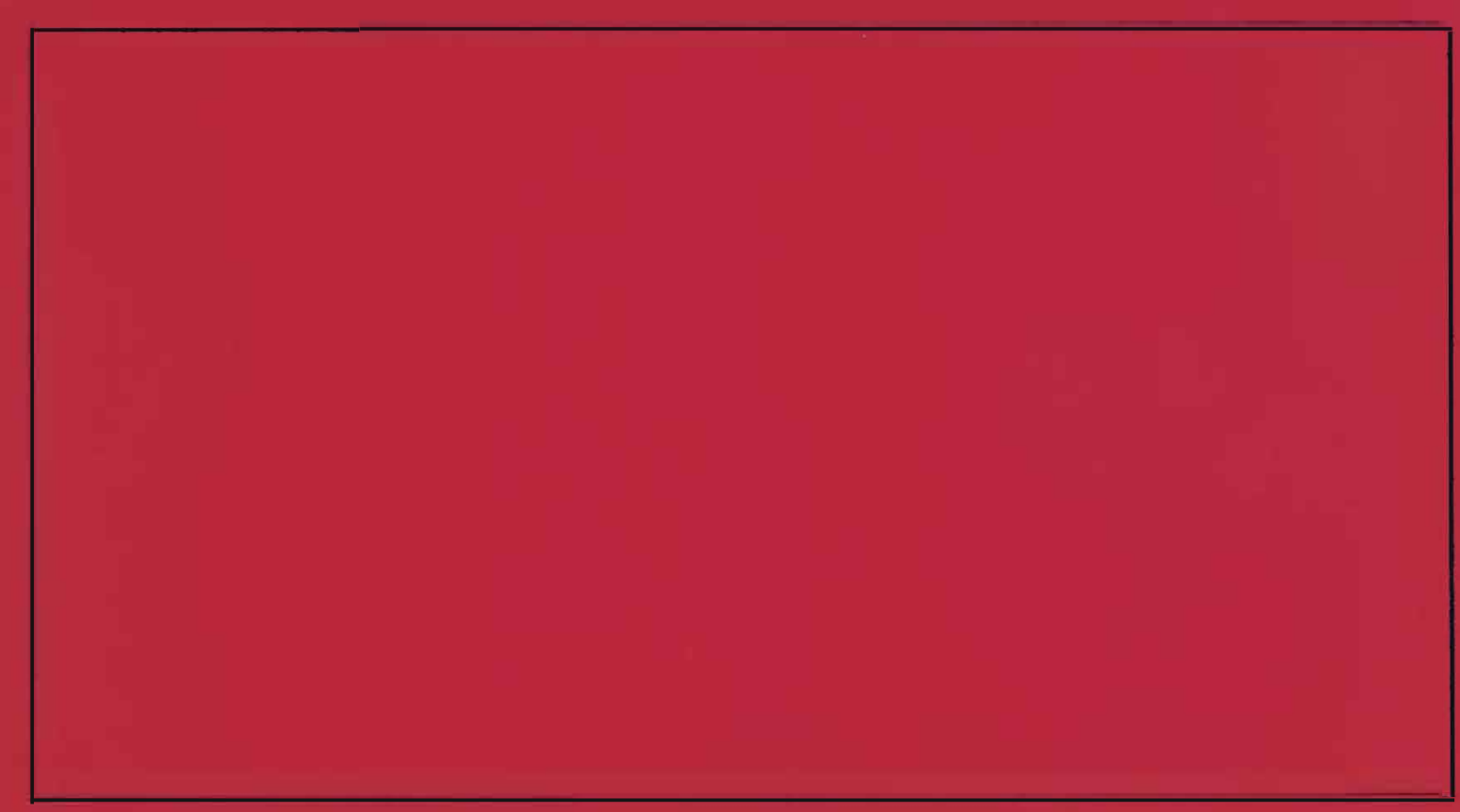

INSTITUT DE MATHEMATIQUES ECONOMIQUES

UNIVERSITE DE DIJON

FACULTE DE SCIENCE ECONOMIQUE ET DE GESTION

4. BOULEVARD GABRIEL -21000 DIJON 


\author{
$\mathrm{N}^{\circ} 46$ \\ PRODUCER'S SPATIAL EQUILIBRIUM \\ WITH A FUZZY CONSTRAINT \\ Claude PONSARD \\ May 1980
}

This paper is to be presented at the Fourth European Congress on Operations Research, Cambridge, England, July 22-25, 1980.

The author is professor at the University of Dijon and Director of the Institut de Mathématiques Economiques, associated with the Centre National de la Recherche Scientilique (lrance). 
SUMMARY

O. Introduction

1. Production Sets

1.1. The analytical framework

1.2. The set of all technically possible productions

1.3. The fuzzy subset of the efficient productions

2. The Producer's spatial Behaviour

2.1. The spatial price system

2.2. The producer's choice criterion

3 . The Producer's spatial Equilibrium

3.1. Objective and constraint in a fuzzy context

3.2. The producer's fuzzy economic calculation

4. Conclusion

5. Bibliography 


\section{O. INTRODUCTION}

0.1. The classical theory of the producer's equilibrium rests on two sets of particularly restrictive hypotheses. First it is implicitely assumed that all inputs and outputs are located in a single place where the producer is also implanted and where the production is carried out.

Next it is assumed that the producer follows a precise behaviour pattern, by this we mean that the producer has complete information concerning the conditions of his productive activity and he has perfect command over both the set of inputs and the set of outputs; he realises the maximum profit allowed by the technological constraint which limits his possible actions and by the given price system.

0.2. The aim of this study is to discard these two families of hypotheses relating first to the economic space of goods and of the agent and second to the precision of the economic agent's behaviour.

All inputs are not assumed to be available and accessible at the production place. They are scattered around the supply space and are transportable. If an input is both necessary and untransportable, then its location determines the implantation of the production unit. Similarly the outputs are not all sold at the place of production. If they are transportable, they are distributed in a demand space; if not, they have to be used on the spot. The transport costs of inputs and outputs are calculated from the

The author wishes to thank Mrs Margaret CHEVAILLIER who translated this paper into English and Mr Michel PREVOT (IMli) whose suggestions cnabled him to improve some demonstrations, in particular those developed in paragraphs 3.2 .4 and 3.2.5. of course, the author alone is responsible for any shortcomings or possible mistakes in the text. (C.P.) 
location of the production unit, which is given.

Moreover the producer's spatial behaviour is imprecise. The economic agent has a utility function for profit such that for a given spatial price system, a greater or lesser degree of satisfaction is associated with any production. Furthermore, the producer has only imperfect control over the production process. Even if the physical combination of the inputs used to obtain one or several given outputs is optimal with respect to the state of technology, it usually yields a result which is short of the maximal product to a greater or lesser degree.

0.3. This leads us to elaborate a theory of the producer operating in an imprecise context, formalised with the help of instruments from the theory of fuzzy subsets [4] [9]. In order to avoid any ambiguity, it must be pointed out that reliability is a particular case of imprecision; it has been demonstrated rigorously that randomness is a particular case of fuzziness [4][7][8][12].

This study will obey the following plan. First the production sets will be described. Having defined the analytical framework, the main characteristics of the set of all possible productions and the fuzzy efficient productions subset will be given.

Then the producer's spatial behaviour will be studied. The environment in which he acts is described by a given spatial price system and his action obeys a choice criterion.

We will then have all the necessary elements to enable us to define the producer's spatial equilibrium in an imprecisc context. The problem is to determine an optimal fuzzy supply, that is, the supply which maximises the fuzzy utility of profit under a fuzzy technological constraint and with respect to a given spatial price system. 
0.4. Notation: Ordinary mathematical concepts (non fuzzy) are underlined, whereas fuzzy concepts are not. For instance, $H \subset \underline{Z}$ is read: $H$ is a fuzzy subset of the referential $\underline{Z}$. For simplification's sake, this convention is only applied to sets and not to their elements. No ambiguity is possible. 


\section{PRODUCTION SETS}

1.1. The Analytical Framework

1.1.1. The producer is described in a general way: his type of activity and the organisational structure of his production unit are disregarded. He is an individual or a collective agent who selects and carries out a production plan, ie. a combination of inputs with a view to obtaining a set of outputs.

1.1.2. The producer's space is characterized by the location of his production unit, by the inputs supply space and the outputs demand space.

(1.1.2.1) The location and the dimension of the production are given in one place indexed 1. By dimension we mean the existing production capacity. It is fixed. The analysis is limited to the short term.

(1.1.2.2) The inputs supply space peculiar to the producer is characterized by the datum of $\mathrm{p}$ places, indexed $\mathrm{r}$, with $r=1, \ldots, p$ where the inputs are accessible and available. The production process requires $n$ inputs, denoted by $\underline{Y}_{i}^{r}$ with $i=1, \ldots$, n and $r=1, \ldots, p$. A place can supply one or several inputs.

Since this is a short term analysis, the set of inputs does not include fixed assets, their location and size are given.

(1.1.2.3) The outputs demand space is defined by the datum of $(q-p)$ places, indexed $r=p+1, \ldots, q$, where these outputs can be sold.

The production process generates $(m-n)$ outputs, denoted by $\underline{Y}_{i}^{r}$, with $i=n+1, \ldots, m$ and $r=p+1, \ldots, q$.

A placc can demand one or several outputs.

1.1.3. Hence there is a finite number of distinct goods, inputs and outputs, which define the set of located goods peculiar to the producer, denoted by $\underline{Y}$, with $\underline{Y}=\left\{\underline{Y}_{i}^{\mathrm{r}}\right\}$, $\mathrm{i}=1, \ldots, \mathrm{m}$ and $\mathrm{r}=1, \ldots, \mathrm{q}$. 
If any one locality supplies (resp.demands) more than one input (resp.more than one output) it will be represented by the same number of points as inputs supplied (resp.outputs demanded). Similarly if any one locality is both an inputs supply place and an outputs demand place, it is represented by the same number of points as supply or demand functions fulfilled.

The goods space peculiar to the producer thus has $n \cdot p+(m-n)(q-p)=n(2 p-q)+m(q-p)=k$ coordinates and $\underline{Y}$ is included in $\mathbb{R}^{\mathrm{k}}$.

If $\mathbb{R}^{1}$, with $1 \geqslant k$, designates the goods space peculiar to the whole economy, it is clear that $\underline{Y}$ is generally contained in a subspace of $\mathbb{R}^{1}$ which has a small number of dimensions.

It is customary in modern producer analysis to represent input quantities by negative real numbers and output quantities by positive real numbers.

Using these notations, a production denoted by $y$ is represented by a point of $\mathbb{R}^{k}$. We have $y=\left(y_{i}^{r}\right)$ with $i=1, \ldots, m$ and $r=i, \ldots, q$.

1.2. The set of all technically possible productions 1.2.1. A production $y_{\text {may }}$ or may not be technically possible by the producer. The subset, denoted by $\underline{Z}$ of the possible productions included in $\underline{Y}$ is called the production set peculiar to the producer. An element $y$ of $\underline{Z}$ is therefore called the producer's supply. 1.2.2. The following assumptions can be laid down on $\underline{Z}$, since they are well-known no comment will be made about them. [1] [2] [3]

$(1.2 .2 .1)$ continuity: $\underline{Z}$ is closed

(1.2.2.2) impossibility of free production: an output cannot be produced without using an input: $\left[\mathbb{I R}^{\mathrm{k}+}-(\vec{O})\right] \cap \underline{Z}=\varnothing$ 
(1.2.2.3) possibility of inactivity: the element $\overrightarrow{0}$ of $\mathbb{R}^{k}$ is an element of $\underline{Z}$.

(1.2.2.4) possibility of the production of wastes: $\mathrm{R}^{\mathrm{k}-} \subset \underline{Z}$

$(1.2 .2 .5)$ convexity: $\underline{Z}$ is a convex subset of $\mathbb{R}^{\mathrm{k}}$

(1.2.2.6) additivity: $\forall y \in \underline{z}, \forall y^{\prime} \in \underline{z},\left(y+y^{\prime}\right) \in \underline{z}$

(1.2.2.7) homogeneity: $\underline{Z}$ is a cone of the summit 0 .

(1.2.2.8) divisibility: if $y \in \underline{Z}, \forall a \in[0,1]$, ay $\in \underline{z}$.

These hypotheses do not all intervene at the

same level of the analysis. They are not all independent. Finally, hypothesis $(1.2 .2 .5)$ calls for greater attention and because of its specific application in spatial analysis the whole of paragraph 2.1 .4 is devoted to it.

1.3. The fuzzy subset of the efficient productions

1.3.1. In traditional theory, a preorder on $\mathbb{R}^{\mathrm{k}}$ denoted by is defined by:

$$
\forall y=\left(y_{i}^{r}\right), y \in \underline{z}, \forall y^{\prime}=\left(y_{i}^{\prime}\right), y^{\prime} \in \underline{z}:
$$

$\left[y \geqslant y^{\prime} \Leftrightarrow\left(y_{i}^{r}\right) \geqslant\left(y_{i}^{\prime r}\right), y \neq y^{\prime}, \forall i=1, \ldots, m\right.$,

(with at least one strict inequality), and a production $y$ is said to be efficient in $\underline{Z}$ if and only if: $\forall y^{\prime} \in \mathbb{R}^{k},\left(y^{\prime} \geqslant y\right) \Rightarrow\left(y^{\prime} \notin \underline{z}\right)$.

The efficient productions set in $\underline{Z}$ is denoted by H. We show that hypothesis $(1.2 .2 .1)$ is a necessary condition and that hypotheses (1.2.2.2) and (1.2.2.3) are jointly a sufficient condition to the existence of an efficient production in $\underline{Z}$ [1] [2].

1.3.2. The fundamental point under discussion here is that the traditional theory of the producer holds true for the limiting casc in which (1) all the inputs have, in a productive combination, a maximal technical efficiency and (2) alone determine the quantities of outputs obtained. llowever, this is not generally the case. 
On the one hand, the efficiency of an input is relative and depends on several technical, institutional or other factors which are 1 inked to its state and the conditions of its use. These factors cannot be weighted. Hence each input's contribution to the product only rarely reaches the maximal theoretical limits made possible by the prevailing state of technology.

On the other hand, it is generally impossible to take all the factors which influence the production into account such that, even if their technical efficiency is maximal, the inputs under consideration do not alone determine the quantity of outputs. This is true of free factors, incontrolable factors and fixed factors. Their action on the production process is sufficient to make the technical efficiency of well-controlled inputs imprecise.

For all these reasons, the result of a production process is by nature imprecise. It follows that a technically possible production is more or less efficient. It is not advisable to partition the set of all possible productions into two classes: the efficient productions and the inefficient productions. As soon as at least one of the inputs does not have a maximal technical efficiency and/or as soon as at least one non-controlled input has an influence on the output quantities, the result of the productive combination is fuzzy. For a given combination the quantites of outputs obtained depend on the degree of efficiency of each of the controlled inputs and on the action of the factors which remain beyond the producer's control.

1.3.3. Formally, a set of more or less efficient productions is defined as a fuzzy subset of $\underline{z}$, denoted by $H$. Its elements have a membership function such that:

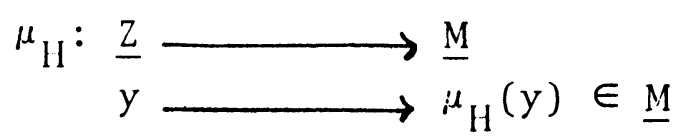


where $\underline{M}$ is a preordered and bounded membership set and where $\mu_{H}(y)$ is called the degree of membership of the element $y$ of $\underline{z}$ to the fuzzy subset $H$.

We choose $\underline{M}=[0,1]$ and $H$ is thus a fuzzy subset in Zadeh's sense [11].

Let us assume that: $\mu_{H}(y)=1$ if the technical efficiency of all the inputs is maximal and if all the inputs are well controlled,

$$
\mu_{H}(y)=0 \text { if } y \in \mathbb{R}^{k-} \subset \underline{z}
$$

ie. in the case of the production of wastes (hypothesis $(1.2 .2 .4))$.

$$
\text { and } \left.\mu_{\mathrm{H}}(y) \in\right] 0,1[
$$

if the technical efficiency of the production process lies between these extreme values. Hence $H$ is a fuzzy subset of $\underline{Z}$ such that:

$$
\mathrm{H}=\left\{y, \mu_{\mathrm{H}} ; \forall y \in \underline{z}, \mu_{\mathrm{H}}(y) \in[0,1]\right\} \text {. }
$$

(1.3.3.1) Remark: In the particular case when the membership set $M$ is the set $\{0,1\}$ we come back to the classical theory of the producer.

(1.3.3.2) The membership functions are determined by purely technical considerations. The fuzziness is objective. 
2. THL PRODUCER'S SPATIAL BEHAVIOUR

2.1. The spatial price system

2.1.1.The spatial price system is given (competition hypothesis) and peculiar to the producer. Such a system is defined by the F.O.B. prices of inputs, the C.I.F. prices of inputs, the F.O.B. prices of outputs, the C.I.F. prices of outputs and the transport prices. A price system is spatial and peculiar to a producer in the sense that it depends on the location of the production unit and on the distances which separate the unit from the inputs supply places and the outputs demand places.

2.1.2. Let ${ }^{f} p_{i}$, with $i=1, \ldots, n$ and $r=1, \ldots, p$, be the F.O.B. unit prices of inputs $y_{i}^{r}$ supplied to the places indexed $r$.

For any $i=1, \ldots, n$ and for any $r=1, \ldots, p$ the unit transport prices of the inputs $y_{i}^{r}$ from the places $r$ to place 1 (the location of the production unit) equal the expenditures necessary for the delivery of those inputs units (transport costs proper, insurance premiums, costs of different operations). These unit transport prices are denoted by $t_{i}^{r \rightarrow 1}$.

The C.I.F. unit prices of the inputs $y_{i}^{r}$ at the locality of the production unit are equal to:

$$
{ }^{f} p_{i}^{r}+t_{i}^{r \rightarrow 1}={ }^{c} p_{i}^{r} \quad \text { with } i=1, \ldots, n \text { and } r=1, \ldots, p
$$

The outputsprices are defined in the same way. Let ${ }^{f} p_{i}^{r}$, with $i=n+1, \ldots, m$ and $r=1$ be the F.O.B. unit prices of the outputs at the production place. For any $i=n+1, \ldots, m$ and for any $r=p+1, \ldots, q$ the unit transport prices of the outputs $y_{i}^{r}$ from the place indexed 1 to the places of demand, indexed $r$, are denoted by $t_{i} \rightarrow_{r}$. The C.I.F. unit prices of outputs. $y_{i}^{r}$ to the places of demand are equal to:

$$
s_{p_{i}}^{r}+t_{i}^{1 \rightarrow r}=c_{p}^{r} \text { with } i=n+1, \ldots, m \text { and } r=p+1, \ldots, q \text {. }
$$


2.1.3. Under competition, the transport price is borne by the buyer who, by assumption, has no means of passing the cost onto the seller. The unit prices paid by the producer for the purchase of the inputs $y_{i}^{r}$ are therefore equal to ${ }^{c} p_{i}^{r}$, with $i=1, \ldots, n$ and $r=1, \ldots, p$.

On the other hand, the net unit prices received by the producer for the sale of the outputs $y_{i}^{r}$ are equal to: $c_{p_{i}}^{r}-t_{i}^{1 \rightarrow r}=f_{p_{i}}^{r}$, with $i=n+1, \ldots, m$ and $r=p+1, \ldots, q$.

Finally, a real number, its price for the producer, is associated to each element of the set of commodities peculiar to the producer $\underline{Y} \cdot \mathrm{p}$ denotes a spatial price system for the producer. Such a system is a k-uple

$$
p=\left({ }^{c} p_{1}^{1}, \ldots{ }^{c} p_{i}^{r}, \ldots,{ }^{c} p_{n}^{p},{ }^{f} p_{n+1}^{p+1}, \ldots,{ }^{f} p_{i}^{r}, \ldots,{ }^{f} p_{m}^{q}\right) .
$$

The price of commodity is positive (scarcity and utility) zero (gratuity) or negative (disutility).

2.1.4. We note that since the prices ${ }^{c} p_{i}^{r}$ with $i=n+1, \ldots, m$ and $r=p+1, \ldots, q$, do not diminish with the increasing distances between the locality of the production unit and those of the buyers of the outputs, all other things being equal, the demand for these outputs tends towards zero when the prices tend towards sufficiently high values. Strictly, competition is never complete in space.

A quite common mistake consists in adding transport costs - when they are taken into account - to the production costs with out distinguishing their particuliar effects. Yet transport prices do have an effect on the optimal volumes of production and on producer's profits such that hypothesis $(1.2 .2 .5)$ proves to be less restrictive in the framework of producer's spatial theory than in that of classical theory [5]. 
2.2. The producer's choice criterion

2.2.1. In classical theory, the producer's choice criterion is the maximum profit where profit is defined as total

receipts less total expenditure. According to the institutienal characteristics of the production unit, the maximum profit objective corresponds to fulfillment of the producer's own particular self-interest or to the satisfaction of an indicator of social efficiency.

In this framework, the producer's economic calculation boils down to the maximisation of a profit function under a strict technological constraint.

Given a spatial price system $p$ and a production $y$ in $\underline{Z}$, the producer's profit is by definition the interior product py, with:

$$
\text { py }=\sum_{i=n+1}^{m} \sum_{r=p+1}^{q} f_{p} p_{i}^{r} y_{i}^{r}-\sum_{i=1}^{n} \sum_{r=1}^{p} c_{p}^{r} y_{i}^{r}
$$

The producer must choose a production y such that his profit is maximum, ic. a distribution of his located inputs and his located outputs which maximises py under the technological constraint. Such an optimal production is called an equilibrium production with respect to a spatial price system.

2.2.2. This classical conception holds true in the limiting case when the producer's behaviour is precise, that is when the maximum profit criterion dictates his behaviour and when, moreover, the technological constraint is rigid. These two assumptions imply perfect and complete information about the arguments of the profit function and the technological constraint.

(2.2.2.1) Cicnerally, the producer's behaviour is, like any human behaviour, imprecise.

The concept of profit aggregates all the points of view the producer has on the results of his productive activity. However these points of view are numerous and not necessarily 
compatible. It follows that a given profit is of greater or lesser utility to the agent. In particular, the greatest profit may prove less useful to the producer than a lower profit if the former doesn't reconcile so well as the latter the conflicting points of view concerning the results of the concern.

depends on $y$.

Let $\underline{\mathrm{p}}=\mathrm{py}$. As $\mathrm{p}$ is given and constant:.., $\underline{\mathrm{p}}$ on $1 \mathrm{y}$

We build the function, denoted by $\mu_{p}$, called imprecise profit utility function which makes an element of the interval $[0,1]$ correspond to each element of the set $\underline{Z}$.

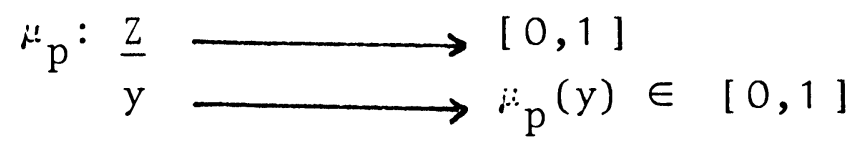

where $\mu_{p}(y)$ is called the degree of membership of the element $y$ of $\underline{Z}$ to the fuzzy subset $P$.

Let us assume that: $:{ }_{\mathrm{p}}(y)=0$ if the inactivity assumption $(1.2 .2 .3)$ is verified

cases.

$$
\text { and } \left.\left.: \mu_{p}(y) \in\right] 0,1\right] \text { in the other }
$$

The function $\mu_{p}(y)$ depends directly on the result of the aggregation of the points of view expressed about the products of the concern.

At the limit, we have $\mu_{p}(y)=1$ for a production $y$ which reconciles the best these points of view. The amount of profit associated with it yields the maximal utility of profit. We are back to the classical hypothesis if the utility of profit is maximal for the maximum amount of profit. llence $P$ is a fuzzy subset of $\underline{Z}$ such that:

$$
P=\left\{y, r_{p} ; \forall y \in \underline{z}, H_{p}(y) \in[0,1]\right\} \text {. }
$$

(2.2.2.2) The membership functions are determined by personal views which the producer synthesizes. The fuzziness is subjective. 
3. THE PRODUCER'S SPATIAL EQUILIBRIUM

3.1. Objective and constraint in a fuzzy context.

3.1.1. To solve the problem of the maximisation of the producer's profit under a technological constraint, modern classical theory uses Minkowski's theorem which states that a hyperplane I exists which passes through $y$ and leaves the whole of $\underline{Z}$ on one side, if and only if a point $y$ of $\mathbb{R}^{k}$ is not interior to the convex subset $\underline{Z}$ of $\mathbf{R}^{\mathrm{k}}$ [1] [3].

For $p \neq 0, y \in \underline{H}$, the convex part $\underline{Z}$ is contained in the closed half-space under the hyperplane $I$, passing through $y$, orthogonal to $\mathrm{p}$. The intersection of $\underline{I}$ and $\underline{H}$ gives the optimal productions set. An equilibrium production is a point or a set of points in the efficient productions set (subset of the frontier of the set of all possible productions) which depend on the price system [2].

3.1.2. Minkowski'stheorem does not apply when the production sets are fuzzy subsets. The latter are not disjointed and it is impossible to find a separating hyperplane.

The technological constraint and the objective function must therefore be defined in a fuzzy context, and the problem of the maximisation of the fuzzy utility of profit must be solved under the fuzzy technological constraint. We can then verify that the producer's fuzzy economic calculation comes down to a fuzzy mathematical programming. (3.1.2.1) In paragraphs 1.3.2. and 1.3.3. we showed that any point $y$ of $\underline{Z}$ is a more or less efficient production and we defined the fuzzy subset $H$ of $\underline{Z}$ each element of which is equipped with a function which expresses its degree of membership to this subset of more or less efficient productions.

This conception widens the sphere of search for the equilibrium production, that is for the producer's optimal supply, since that supply now belongs to any space bounded 
by the frontier of the set of all possible productions, and no longer, as in the limiting case examined in classical theory, to a subset of that frontier.

Formally, the fuzzy subset $H$ therefore plays the role of a constraint in the producer's fuzzy economic calculation.

(3.1.2.2.) In paragraph (2.2.2.1) we admitted that, placed in a imprecise universe, the producer's aim is to maximise the utility he gets from the profit realized. In a particular case, this objective coincides with that of maximum profit.

We have defined the fuzzy subset $P$ of $\underline{Z}$ whose elements are equipped with functions which translate their respective degrees of membership to this productionssubset having profits associated with it which are more or less useful to the producer.

3.2. The producer's fuzzy economic calculation

3.2.1. The producer's equilibrium is attained for an optimal supply with respect to the spatial price system $p$ and the fuzzy technological constraint $H$, with reference to its utility for profit, that is the fuzzy objective P.

$S$ designates the producer's optimal supply which maximises the utility of profit for the producer over the fuzzy subset of the more or less efficient productions. Hence, by definition, $S$ is a fuzzy subset of $\underline{Z}$ such that: $S=P \cap H$ and the producer's fuzzy economic calculation is an example of a Fuzzy Mathematical Programming (F.M.P.) [6][10] .

Indeed, the supply may be interpreted as a fuzzy decision. It is the intersection of the fuzzy subsets $P$ ( the set of all productions which engender profits judged to be more or less useful) and $H$ (the set of all the more or less technically efficient productions).

The optimal supply has to be determined such that:

$$
\sup _{y \in \underline{Z}} \cdot \mu_{S}(y)=\sup _{y \in \underline{Z}}\left[\mu_{p}(y) \Lambda \mu_{H}(y)\right] \text {. }
$$


The solution employs the results of the F.M.P. theory, nevertheless certain technical details of its presentation have been modified and adapted to the economic problem under consideration.

3.2.2. Proposition:

$$
\sup _{y \in \underline{Z}} \mu_{S}(y)=\sup _{u \in[0,1]}\left[\begin{array}{ll}
a & \left.\Lambda \sup _{y \in \underline{H}_{\alpha}} \mu_{p}(y)\right]
\end{array}\right.
$$

where

$$
\underline{H}_{u}=\left\{y ; y \in \underline{z}, \quad \mu_{H}(y) \geqslant \alpha\right\} \quad \text { with } \alpha \in[0,1] .
$$

(3.2.2.1) Definition:

$\underline{H}_{\alpha}$ is called $\alpha$-cut of the fuzzy subset $H$ of $\underline{Z}$.

The set of $\alpha$-cuts, denoted by $\left\{\underline{H}_{-}\right\} \alpha \in[0,1]$, is a decreasing sequence such as:

and

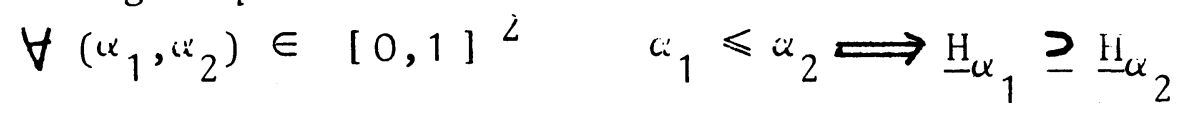

$$
\underline{H}_{\mathrm{O}}=\underline{\mathrm{Z}}
$$

(3.2.2.2) We need the following result, obtained beforehand, called the decomposition theorem:

Let $H \in \mathcal{P}(\underline{Z})$ and $\left\{\underline{H}_{\alpha}\right\}_{\alpha} \in[0,1]$ be its $\alpha$-cuts, then

$$
\mathrm{H}=\underset{\alpha \in[0,1]}{\mathrm{U}} \boldsymbol{\mathrm { U }} \cdot \underline{\mathrm{H}}_{\alpha}
$$

Indeed, $\quad{ }^{\prime} \underline{\mathrm{H}}_{\alpha}(y)=1 \quad$ if $\quad \because{ }_{\mathrm{H}}(y) \geqslant \alpha_{i}$

$$
\because \underline{\mathrm{H}}_{\alpha_{\mathrm{i}}}(\mathrm{y})=0 \quad \text { if } \quad \mu_{\mathrm{H}}(\mathrm{y})<\alpha_{\mathrm{i}}
$$

Hence, the membership function of $\mathrm{H}$ is written:

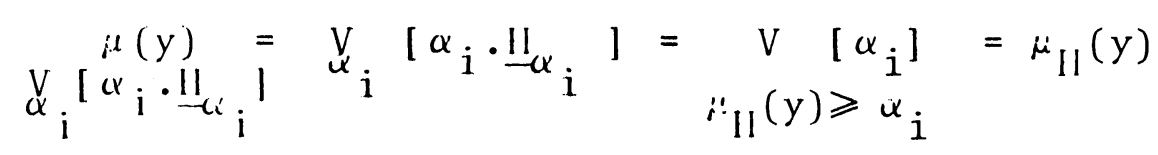

$$
\text { [ Q.L.D.] }
$$

(3.2.2.3) Demonstration of proposition 3.2.2. According to the decomposition theorem we have: 


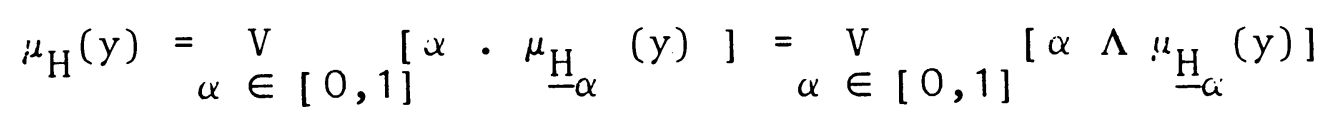

where $\mu_{\underline{H}_{\alpha}}$ is the characteristic function of $\underline{\mathrm{H}}_{\omega}$.

By definition we have:

$$
\begin{aligned}
& r_{\mathrm{S}}(\mathrm{y})=\mu_{\mathrm{P}}(\mathrm{y}) \Lambda \mu_{\mathrm{H}}(\mathrm{y}) \\
& =\mu_{\mathrm{P}}(\mathrm{y}) \quad \Lambda\left[\underset{\alpha \in[0,1]}{\mathrm{V}}\left(\alpha \mu_{\underline{H}_{x}}(y)\right)\right] \text { (property of } \underline{H}_{\alpha} \text { ) }
\end{aligned}
$$

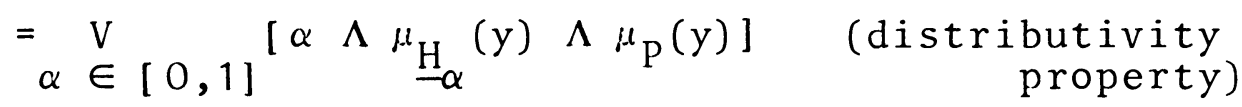

Whence it follows that:

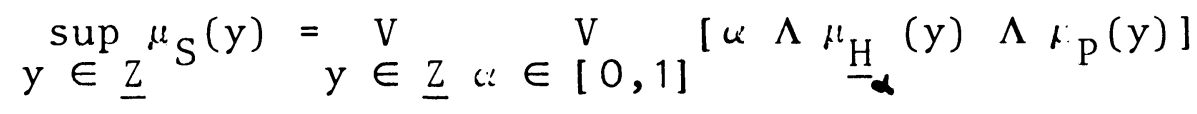

$$
\begin{aligned}
& =\underset{\alpha \in[0,1] y \in \underline{z}^{V}}{V}\left[\begin{array}{ll}
\alpha i_{H_{\alpha}} & \left.(y) \Lambda \mu_{P}(y)\right]
\end{array}\right. \\
& \text { (associativity and commutativity } \\
& \text { properties) }
\end{aligned}
$$

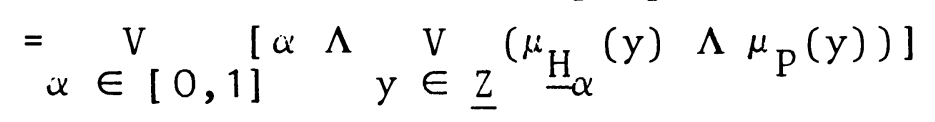

$$
\begin{aligned}
& \text { (because } \alpha \text { is independent of } y \text { ) }
\end{aligned}
$$

By partitioning $\underline{H}_{\alpha}$ we have:

$$
\begin{aligned}
& \underset{y \in \underline{Z}}{\mathrm{~V}} \quad\left[\ddot{\mu}_{\mathrm{H}^{\prime}}(y) \Lambda \mu_{\mathrm{P}}(y)\right]=\underset{y \in \underline{H}_{\alpha}}{ }\left[\underline{\mu}_{a}(y) \Lambda \mu_{\mathrm{P}}(y)\right] \mathrm{V} \\
& \left.\mathrm{y} \stackrel{\mathrm{V}}{\notin \underline{\mathrm{H}}_{\alpha}} \underline{\mu}_{\alpha}(\mathrm{y}) \Lambda \cdot \mu_{\mathrm{P}}(\mathrm{y})\right]
\end{aligned}
$$

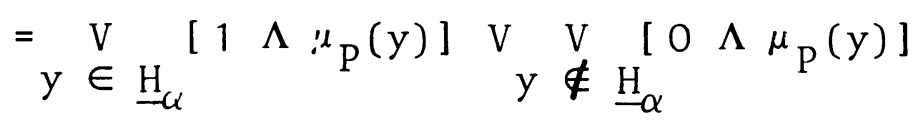

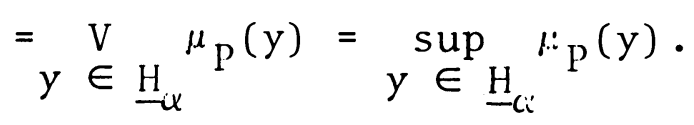

Therefore we can write:

$$
\sup _{y \in \underline{Z}}{ }^{\mu} S_{S}(y)=\sup _{\alpha \in[0,1]}\left[\alpha \Lambda \sup _{y \in \underline{H}_{\alpha}} \mu_{P}(y)\right]
$$

(3.2.2.4) Remark:

[Q.E.D.]

This proposition reduces the problem of finding the fuzzy optimal supply to that of finding the extremum of a scalar function [6]. 
3.2.3. Let us now introduce a function $\varphi$ such that:

$$
\begin{aligned}
& \varphi:[0,1] \longmapsto[0,1] \\
& \alpha \longmapsto \varphi(\alpha)=\sup _{y \in \underline{H}_{c}} \mu_{p}(y)
\end{aligned}
$$

and a function $\Psi$ such that:

$$
\Psi:[0,1]
$$

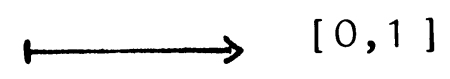

$\alpha$

$$
\Psi(\alpha)=\alpha \Lambda \varphi(\alpha)
$$

Then: $\sup _{y \in \underline{Z}} i_{s}(y)=\sup _{u^{\prime} \in[0,1]} \Psi(\alpha)$

$$
\text { (proposition 3.2.2.) }
$$

(3.2.3.1.) Properties of the function $\varphi$ :

$$
(3.2 .3 \cdot 1.1 .): \varphi(0)=\sup _{y \in \underline{Z}} \mu p(y)
$$

This is obvious, since $\underline{\mathrm{H}}_{\mathrm{O}}=\underline{\mathrm{Z}}$

$$
(3.2 .3 .1 .2 .): \quad u \leqslant i \Longrightarrow \varphi(\alpha) \geqslant \varphi(j) \text {. }
$$

Indeed, according to the decomposition theorem:

$$
\begin{array}{r}
\alpha \leqslant \beta \Longrightarrow \underline{H}_{\beta} \subseteq \underline{H}_{\alpha} \quad \text { and thus } \\
\varphi(\beta)=\sup _{y \in \underline{H}_{\beta}} \mu_{p}(y) \leqslant \sup _{y \in \underline{H}_{\alpha}} \mu^{\prime} p(y)=\varphi(\kappa)
\end{array}
$$

[ Q.E.D.]

(3.2.3.2.) Proposition: If $\varphi$ is continuous and decreasing over the interval $[0,1]$ then:

(i) $\varphi$ has a fixed point, that is:

$$
\exists \bar{c} \in[0,1] / \varphi(\bar{u})=\bar{u} \text { and } \bar{u}=\sup _{y \in \underline{H}_{\bar{u}}^{\mu}}{ }^{\mu} p(y) \text {. }
$$

Indeed, first we show that any continuous and decreasing application of a set in itself implies a fixed point.

Let $\varphi(\alpha)-\alpha=f(\alpha)$. This function is continuous.

We have : $f(0)=\varphi(0)>0$

$$
f(1)=\varphi(1)-1 \leqslant 0
$$

and in order to verify the existence of a fixed point we merely have to apply the theorem of intermediate values. Then, according to the continuity of $\varphi$ :

$$
\begin{aligned}
& \forall c_{0} \in[0,1], a_{n} \longrightarrow \alpha_{0} \Longrightarrow \lim _{n \rightarrow \infty}\left[\sup _{y \in \underline{H}_{n}} i(y)\right]=\sup _{y \in \underline{H}_{-}} \mu_{p}(y) . \\
& \text { [Q.E.D.] }
\end{aligned}
$$


(ii) $\sup _{y \in \underline{Z}} \mu_{S}(y)=\bar{\alpha}$

Indeed, according to proposition 3.2.2.:

$$
\sup _{y \in \underline{z}} \underline{i l}_{S}(y)=\sup _{\alpha \in[0,1]}\left[\alpha \sup _{y \in \underline{H}_{\alpha}}{ }_{p} p(y)\right]
$$

$=\sup _{\alpha \in[0,1]} \Psi(\alpha)$

[from the definition of $\Psi(\alpha)$ ]

Moreover, $\Psi(\bar{\alpha})=\bar{\alpha} \Lambda \quad \varphi(\bar{\alpha})=\bar{\alpha} \Lambda \bar{\alpha} \quad$ [from (i)]

$$
=\bar{\alpha}
$$

Let us show that: $\Psi(\alpha) \leqslant \bar{\alpha}$

If $\alpha<\bar{\alpha}$, we have: $\varphi(\alpha) \geqslant \varphi(\bar{\alpha}) \quad$ [from (3.2.3.1.2.)]

$$
\varphi(\alpha) \geqslant \varphi(\bar{\alpha})=\bar{\alpha}>\alpha \quad[\text { from (i)] }
$$

and $\Psi(\alpha)=\alpha \Lambda \varphi(\alpha)=\alpha<\bar{\alpha}=\Psi(\bar{\alpha})$.

If $\alpha \geqslant \bar{\alpha}$, we have: $\varphi(\alpha) \leqslant \varphi(\bar{\alpha})=\bar{\alpha} \leqslant \alpha$

and $\Psi(\alpha)=\alpha \Lambda \varphi(\alpha)=\varphi(\alpha) \leqslant \bar{\alpha}=\Psi(\bar{\alpha})=\bar{\alpha} \Lambda \varphi(\bar{\alpha})=\bar{\alpha}$

In every case : $\Psi(\alpha) \leqslant \Psi(\bar{\alpha})=\bar{\alpha}$

Whence: $\quad \bar{\alpha}=\sup _{y \in \underline{z}_{1}} i^{i l} \mathrm{~s}(y)$

$$
\text { [Q.E.D.] }
$$

(3.2.3.3.) Proposition (3.2.3.2.) shows that the solution to the problem of finding $\sup _{y \in \underline{Z}} \mu_{S}(y)$ amounts to finding $\bar{\alpha}=\sup _{y \in \underline{H}_{\bar{\alpha}}} a_{\mathrm{p}}(y)$.

We know that:

$$
\sup _{y \in \underline{Z}} i_{S}(y)=\sup _{y \in \underline{Z}}\left[\mu_{p}(y) \Lambda i_{H}(y)\right]=\sup _{y \in \operatorname{supp} \underline{H}}\left[l_{p}(y) \Lambda \mu_{H}(y)\right]
$$

where supp $\underline{H}$ stands for the support of $H$, that is the non fuzzy subset such that:

$$
\operatorname{supp} \underline{H}=\left\{y ; \forall y \in \underline{Z}, \quad i_{H}(y)>0\right\} \text {. }
$$

liurthermore, by virtue of propositions (ii) and (i), we have:

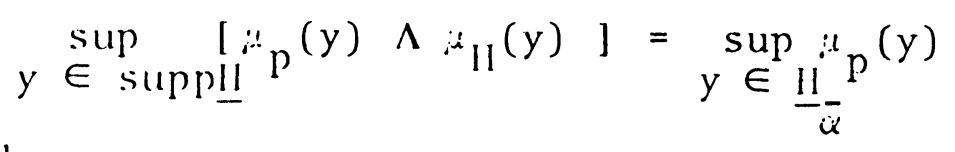

and

$$
\underline{I}_{\bar{\alpha}} \subset \operatorname{supp} \underline{H}, \text { since } \bar{u} \neq 0 \text {. }
$$


In order to calculate this supremum we have to find an appropriate set, denoted by $\underline{A}$, such that:

$\underline{\mathrm{H}}_{\bar{\alpha}} \subset \underline{\mathrm{A}} \subset \operatorname{supp} \underline{\mathrm{H}}$.

(3.2.3.4.) Proposition: $\sup _{y \in \underline{A}} \mu_{p}(y)=\sup _{y \in \underline{Z}} \mu_{s}(y)$,

with $\underline{A}=\left\{y ; y \in \underline{Z}, \mu_{H}(y) \geqslant \mu_{p}(y)\right\}$

We have:

Indeed, first we demonstrate that $\underline{H}_{\bar{\alpha}} \subset \underline{A}$.

$$
\begin{gathered}
\forall y^{\circ} \in \underline{H}_{\bar{\alpha}} \Longrightarrow \mu_{\mathrm{H}}\left(y^{\circ}\right) \geqslant \bar{a}=\sup _{\mathrm{y} \sin _{\bar{\alpha}} \mu_{\mathrm{P}}(y) \geqslant \mu_{\mathrm{P}}\left(\mathrm{y}^{\circ}\right)} \\
\Longrightarrow \mu_{\mathrm{H}}\left(\mathrm{y}^{\circ}\right) \geqslant \mu_{\mathrm{P}}\left(\mathrm{y}^{\circ}\right) \Longrightarrow \mathrm{y}^{\circ} \in \underline{\mathrm{A}} .
\end{gathered}
$$

Furthemore we have:

and

$$
\underline{A}=(\underline{A} \cap \operatorname{supp} \underline{H}) U(\underline{A} \cap(\operatorname{supp} \underline{H})
$$

$$
\begin{aligned}
& \sup \mu_{\mathrm{S}}(\mathrm{y})=\sup \left(\mu_{\mathrm{H}} \Lambda \mu_{\mathrm{P}}\right)(\mathrm{y}) \geqslant \sup \left(\mu_{\mathrm{H}} \Lambda \mu_{\mathrm{P}}\right)(\mathrm{y}) \\
& y \in \underline{Z} \quad y \in \operatorname{supp} \underline{H} \quad y \in \underline{A} \cap \operatorname{supp} \underline{H}
\end{aligned}
$$

But,

$$
\sup _{y \in \underline{\mathrm{A}}}\left(\mu_{\mathrm{H}} \Lambda \mu_{\mathrm{P}}\right)(\mathrm{y})=\max \left[\sup _{\mathrm{A}}\left(\mu_{\mathrm{H}} \Lambda \mu_{\mathrm{P}}\right)(\mathrm{y}), \sup \left(\mu_{\mathrm{H}} \Lambda \mu_{\mathrm{P}}\right)(\mathrm{y})\right]
$$

$$
=\sup \left(\mu_{\mathrm{H}} \Lambda \mu_{\mathrm{P}}\right)(\mathrm{y})
$$

$\underline{\text { A } \cap \operatorname{supp} \underline{H}}$

It follows that:

$$
\begin{aligned}
& \sup _{y \in \underline{Z}_{\mathrm{S}}} \mu_{\mathrm{S}}(y) \geqslant \sup _{\mathrm{y} \underline{\mathrm{A}}^{\mathrm{A}}}\left(\mu_{\mathrm{H}} \Lambda \mu_{\mathrm{P}}\right)(\mathrm{y})=\sup _{\mathrm{y} \in \underline{\mathrm{A}}_{\mathrm{P}}} \mu_{\mathrm{P}}(\mathrm{y}) \geqslant \sup _{\mathrm{y} \in \underline{\mathrm{H}}_{\bar{\alpha}}} \mu_{\mathrm{P}}(\mathrm{y}) \\
& \text { (since } \left.\underline{\mathrm{H}}_{\bar{\alpha}} \subset \underline{\mathrm{A}}\right),
\end{aligned}
$$

and thus:

$$
\sup _{y \in \underline{Z}} \mu_{S}(y)=\sup _{y \in \underline{A}} \mu_{P}(y) \text {. }
$$

3.2.4. We then study the continuity conditions of the function

$$
\varphi\left(a^{i}\right)=\sup _{y \in \underline{H}_{\alpha}} \mu_{P}(y) \text {. }
$$


(3.2.4.1) Definition : A fuzzy subset $H$ is strictly convex if and only if its membership function is strictly quasi-concave, that is, if and only if:

$\forall y \in \underline{z}, \forall y^{\prime} \in \underline{z}, y \neq y^{\prime},\left[\mu_{H}\left(y^{\prime}\right)>\mu_{H}(y)\right] \Longrightarrow$

$$
\forall \lambda \in] 0,1\left[, \mu_{H}\left[\lambda y+(1-\lambda) y^{\prime}\right]>\left[\mu_{H}(y)\right]\right.
$$

(3.2.4.2) Proposition: If $\underline{Z}$ is compact and $\left\{\underline{H}_{\alpha}\right\}$ is closed and if $\mu_{\mathrm{p}}$ is upper semi-continuous, then $\varphi(\alpha)$ is left continuous .

Indeed, let $\left\{\alpha_{i}\right\}$ be the increasing sequence:

$$
\begin{aligned}
& \alpha_{1} \leqslant \ldots \leqslant \alpha_{i} \leqslant \ldots \leqslant \alpha_{n} \longrightarrow \alpha_{0} \\
& \underline{H}_{\alpha_{n}} \subseteq \underline{H}_{\alpha_{n-1}} \subseteq \cdots \subseteq \underline{H}_{\alpha_{i}} \subseteq \cdots \subseteq \underline{H}_{\alpha_{1}}
\end{aligned}
$$

and $\infty$

$$
\bigcap_{i=1}^{\infty}\left(\underline{H}_{\alpha_{i}^{\prime}}\right)=\underline{H}_{\alpha_{0}}
$$

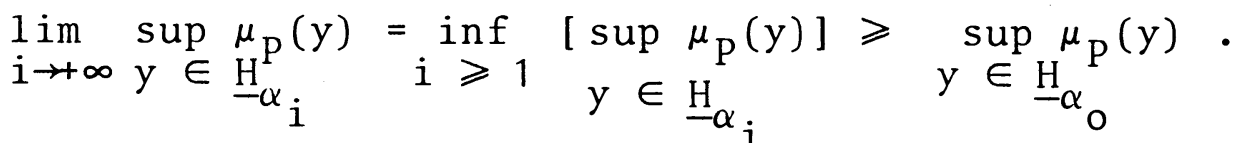

Let us suppose that a strict inequality exists:

$$
\inf _{i} \varphi\left(\alpha_{i}\right)>\varphi\left(\alpha_{0}\right)
$$

such that:

According to the definition of $\varphi$, there exists $(y)_{i} \in \underline{H}_{\alpha}$

$\mu_{\mathrm{P}}(\mathrm{y})_{\mathrm{i}}>\varphi\left(\alpha_{\mathrm{o}}\right)$

Since $(y)_{i} \in \underline{H}_{\alpha}$ and since $\underline{H}_{\alpha_{i}}$ iscompact, a sub-sequence

$(y)_{i_{j}} \rightarrow(y)_{0} \in \underline{H}_{\alpha_{i}}$ exists. Whence it follows that $(y)_{0} \in \underline{H}_{\alpha_{0}}$

Therefore: $\mu_{\mathrm{P}}(\mathrm{y})_{\mathrm{i}_{\mathrm{j}}}>\varphi\left(\alpha_{\mathrm{o}}\right)$.

According to the semi-continuity of $\mu_{\mathrm{P}}$ :

$\mu_{\mathrm{p}}(\mathrm{y})_{\mathrm{O}}>\varphi\left(\alpha_{\mathrm{O}}\right)$.

Whence a contradiction.

[Q.E.D.]

(3.2.4.3) Proposition: If the fuzzy subset $P$ is strictly convex and if $\mu_{\mathrm{P}}$ is continuous, then $\varphi(\alpha)$ is a continuous function. 
It suffices to demonstrate the right continuity. Indeed, let $\left\{\alpha_{i}\right\}$ be the decreasing sequence:

$$
\begin{aligned}
& \alpha_{1} \geqslant \ldots \geqslant \alpha_{i} \geqslant \ldots \geqslant \alpha_{n} \longrightarrow \alpha_{0} \\
& \underline{H}_{\alpha_{1}} \subseteq \underline{H}_{\alpha_{2}} \subseteq \ldots \subseteq \underline{H}_{\alpha_{i}} \subseteq \cdots \subseteq \underline{H}_{\alpha_{n}}
\end{aligned}
$$

and

$$
{\underset{i=1}{U}}_{i}^{\infty} \underline{\mathrm{H}}_{\mathrm{i}}=\underline{\mathrm{H}}_{\alpha_{0}} \text {. }
$$

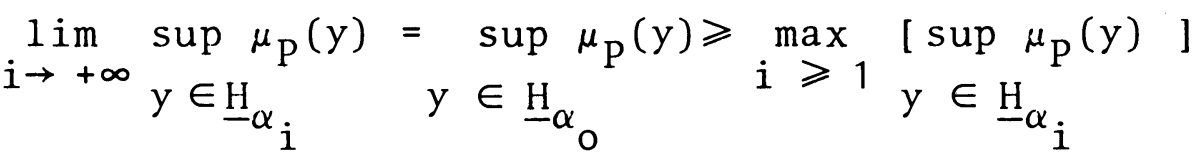

Let us assume that a strict inequality exists.

$\max _{i \geqslant 1} \varphi\left(\alpha_{i}\right)<\varphi\left(\alpha_{0}\right)$

According to this inequality, $\exists y^{\prime} \in \underline{H}_{\alpha_{0}}$ such that:

$$
\begin{gathered}
\mu_{\mathrm{P}}\left(\mathrm{y}^{\prime}\right)>\sup _{\mathrm{P}} \mu_{\mathrm{P}}(\mathrm{y}) \\
y \in \underline{\mathrm{H}}_{\alpha_{i}}
\end{gathered}
$$

By virtue of the strict convexity of $P$, we have:

$\forall y \in \underline{z}, \forall y^{\prime} \in \underline{z}, y \neq y^{\prime}:$

$$
\begin{gathered}
\mu_{\mathrm{P}}\left[\left(1-\frac{1}{n}\right) y^{\prime}+\frac{1}{n} y\right]>\mu_{\mathrm{P}}\left(y^{\prime}\right) \Lambda \mu_{\mathrm{P}}(y)=\alpha_{0} \\
{\left[\left(1-\frac{1}{n}\right) y^{\prime}+\frac{1}{n} y\right]>\alpha_{0} \text { and }\left[\left(1-\frac{1}{n}\right) y^{\prime}+\frac{1}{n} y\right] \longrightarrow y^{\prime}} \\
\mu_{\mathrm{P}}\left[\left(1-\frac{1}{n}\right) y^{\prime}+\frac{1}{n} y\right] \leqslant \sup _{\mathrm{y}} \mu_{\mathrm{P}}(y)<\mu_{\mathrm{P}}\left(y^{\prime}\right) \\
y \in \underline{H}_{\alpha_{i}}
\end{gathered}
$$

According to the continuity of $\mu_{\mathrm{P}}$, we have:

$$
\begin{aligned}
& \mu_{\mathrm{P}}\left(y^{\prime}\right) \leqslant \sup \mu_{\mathrm{P}}(y)<\mu_{\mathrm{P}}\left(\mathrm{y}^{\prime}\right) \\
& y \in \stackrel{H}{-}_{i}
\end{aligned}
$$

We have a contradiction.

[ Q.E.D.]

3.2.5. Finally, it remains to study the unicity conditions of the solution. 
(3.2.5.1.) Lemma 1. If $\left\{\underline{H}_{\alpha}\right\}$ is closed, if $\mu_{\mathrm{P}}$ is strongly quasi-concave and if, $\forall y \in \underline{H}_{\alpha}, \mu_{\mathrm{P}}(y) \neq 1$, the point $\mathrm{y}^{\star}$, such that $\mu_{\mathrm{P}}\left(\mathrm{y}^{\star}\right)=\sup _{\mathrm{y} \in \underline{\mathrm{H}}_{\alpha}} \mu_{\mathrm{P}}(\mathrm{y})$ is an element of the frontier of $\underline{\mathrm{H}}_{\alpha}$, denoted by $\underline{\mathrm{H}}_{\alpha}$.

Indeed, let us suppose that: $y^{\star} \in \stackrel{\circ}{\mathrm{H}}_{\alpha}$, where $\stackrel{\AA}{A}_{\alpha}$ is the interior of $\underline{H}_{\alpha}$, and we shall demonstrate that we end up with a contradiction.

Let us suppose $y_{1}$ such that $\mu_{P}\left(y_{1}\right)=1$. By assumption $\mathrm{y}_{1} \notin \underline{H}_{\alpha}$. Since $\underline{Z}$ is convex, and according to the"passing through customs" theorem, $\exists y^{\prime}$ such that $y^{\prime}=\lambda y_{1}+(1-\lambda) y^{\star}$ and $\mathrm{y}^{\prime} \in \stackrel{\hbar}{-}_{\alpha} \subseteq \underline{H}_{\alpha}$ because $\underline{H}_{\alpha}$ is closed(1).

Since $\mu_{\mathrm{P}}$ is strongly quasi-concave, we have:

$$
\mu_{\mathrm{P}}\left(\mathrm{y}^{\prime}\right)>\min \left[\mu_{\mathrm{P}}\left(\mathrm{y}^{\star}\right), \mu_{\mathrm{P}}\left(\mathrm{y}_{1}\right)\right]=\mu_{\mathrm{P}}\left(\mathrm{y}^{\star}\right) \text {, }
$$

which contradicts the'definition of $y^{\star}$.

(3.2.5.2.) Lemma 2: Any element $y$ of $\underline{H}_{\alpha}$ belongs to the interior of $\underline{H}_{\alpha-\Delta \alpha}$, denoted by $\stackrel{\circ}{H}_{\alpha-\Delta}, \quad \forall \Delta \alpha>0$.

In other terms: $\underline{\mathrm{H}}_{\alpha} \subseteq \underline{\mathrm{H}}_{\alpha-\Delta \alpha}, \forall \Delta \alpha>0$.

Indeed, since $\mu_{H}(y)$ is continuous on $\underline{z}, \quad y \in \underline{H}_{\alpha}$, $\forall \epsilon>0, \quad \eta$ exists such that: distance $\left(y, y_{1}\right)^{2}=\mathrm{d}\left(y, y_{1}\right)^{-\alpha}<\eta$

$$
\Longrightarrow\left|\mu_{\mathrm{H}}(y)-\mu_{\mathrm{H}}\left(y_{1}\right)\right|<\epsilon \quad \text {. }
$$

Let us put $\Delta \alpha=\epsilon$

We have the following inequalities:

and :

$$
\mu_{\mathrm{H}}(\mathrm{y})-\Delta \alpha<\mu_{\mathrm{H}}\left(\mathrm{y}_{1}\right)<\mu_{\mathrm{H}}(\mathrm{y})+\Delta \alpha
$$

$\alpha-\Delta \alpha \leqslant \mu_{\mathrm{II}}(y)-\Delta \alpha$, because by definition

$\mu_{\mathrm{H}}(y) \geqslant \alpha \quad$ in $\underline{H}_{\alpha}$.

Then:

$\alpha-\Delta a_{i} \leqslant \mu_{H}(y)-\Delta \alpha<\mu_{H}\left(y_{1}\right)$

Hence :

$\mathrm{y}_{1} \in \underline{H}_{\mathrm{u}-\Delta a}$

(1) "Passing through customs" Theorem: If a connected subset A included into $E$ intersects with a subset $B$ and its complementary set, then it intersects the frontier of $B$. 
Furthermore, we have: $\forall y \in \underline{H}_{\alpha} \Longrightarrow y \in \underline{H}_{\alpha-\Delta \alpha}$ because $\underline{H}_{\alpha-\Delta \alpha} \supseteq \underline{H}_{\alpha}$. A neighbourhood of $y$ exists, for instance the ball of centre $y$ and of radius $\eta$, contained in $\underline{H}_{\alpha-\Delta \alpha}$ Hence: $y \in \underline{H}_{\alpha-\Delta \alpha}$

[ Q.E.D.]

(3.2.5.3.) Proposition: If $\left\{\underline{\mathrm{H}}_{\alpha}\right\}$ is closed, if $\sup _{\mathrm{y}} \mu_{\mathrm{H}}(\mathrm{y})$ is continuous and if $\mu_{\mathrm{P}}(y)$ is strongly quasi-concave, then $y^{\star}$ is unique.

Indeed from proposition 3.2.2., we have: $\sup _{\alpha \in[0,1]}\left[\alpha \Lambda \sup _{y} \mu_{\mathrm{H}}(y)\right]=\underline{H}_{\alpha}^{\star} \Lambda \mu_{\mathrm{P}}\left(\mathrm{y}^{\star}\right)$.

$$
\text { If } \mathrm{T}=\left\{\begin{array}{c}
-\alpha \\
\mathrm{y} ;
\end{array} \forall \mathrm{y} \in \underline{\mathrm{z}}, \mu_{\mathrm{P}}(\mathrm{y})=\mu_{\mathrm{H}}(\mathrm{y})=1\right\} \neq \emptyset,
$$

then $\mu_{\mathrm{P}}\left(\mathrm{y}^{\star}\right)$ is equal to one and unique.

If $\mathrm{T}=\varnothing$, two values $\mathrm{y}_{1}^{\star}$ and $\mathrm{y}_{2}^{\star}$ are assumed to exist and we demonstrate that we end up with a contradiction.

$$
\alpha_{1}^{\star}>\alpha_{2}^{\star} \text {. Wrom the definition of } y^{\star} \text { and from proposition } 3.2 .2 \text {, , }
$$
we have the relation:

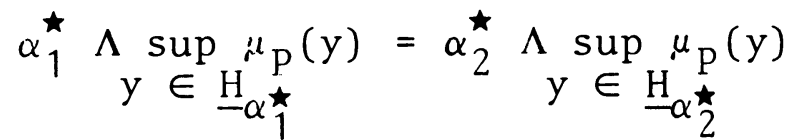

$$
\begin{aligned}
& \text { Since } \alpha_{i}^{\star} \Lambda \sup _{y \in \underline{H}_{\alpha_{i}^{\star}}^{\mu}}{ }_{p}(y) \text { is equal either to } \alpha_{i}^{\star} \text {, or to }
\end{aligned}
$$

sup $\mu_{\mathrm{P}}(\mathrm{y})$, four cases must be distinguished:

$\sup _{y \in{ }_{-}{ }^{\star}}^{\mu}{ }_{i}(y)$

Case $1^{1}: \quad \alpha_{1}^{\star}=\alpha_{2}^{\star}$

Case $2: \alpha_{1}^{\star}=\sup _{y \in \underline{H}_{\alpha}^{\star}} \mu_{p}(y)$

Case $3: \alpha_{2}^{\star}=\sup _{y \in \underline{H}_{1}^{\star}} \mu_{\mathrm{p}}(y)$

Case $4: \sup _{y \in \underline{-\alpha}_{2} \mu_{p}} \mu_{p}(y)=\sup _{y \in \underline{\alpha}_{1}} \mu_{p}(y)$

(3.2.5.3.1.) Case 1 is eliminated by the definition of $\alpha_{1}^{\star}$ and of $a_{2}^{\star}$.

(3.2.5.3.2.) In case 2 , it follows that: 


$$
\alpha_{1}^{\star} \Lambda \sup _{y \in \underline{H}_{\alpha_{1}^{\star}}} \mu_{\mathrm{p}}(y)=\alpha_{1}^{\star}, \text { because } \alpha_{1}^{\star} \leqslant \sup _{y \in \underline{H}_{\alpha_{1}^{\star}}} \mu_{\mathrm{P}}(y)
$$

By assumption: $\alpha_{1}^{\star} \Lambda \sup \mu_{\mathrm{P}}(\mathrm{y})=\alpha_{1}^{\star} \Lambda \sup \mu_{\mathrm{P}}(\mathrm{y})$

$$
y \in \underline{H}_{\alpha}^{\star}{ }_{1}^{\star} \quad y \in \underline{H}_{-\alpha}^{\star}
$$

Yet, from the definition of $\alpha_{2}^{\star}$ :

$$
\begin{array}{cc}
\alpha_{1}^{\star} \Lambda \sup _{y \in \underline{H}_{\alpha_{2}} \mu_{\mathrm{P}}(y)} & \lambda_{2}^{\star} \alpha_{2}^{\star} \Lambda \sup \mu_{\mathrm{P}}(y) \\
y \in \underline{H}_{\alpha}^{\star} & y
\end{array}
$$

which contradicts the definition of $\alpha_{2}^{\star}$.

(3.2.5.3.3.) In case 3, let $\Delta \alpha>0$ such that $\left(\alpha_{1}^{\star}-\Delta \alpha\right)>\alpha_{2}^{\star}$. Since $\alpha_{2}^{\star}$ is a supremum, we have:

$$
\begin{aligned}
& \left(\alpha_{1}^{\star}-\Delta \alpha\right) \Lambda \sup \mu_{\mathrm{P}}(y) \leqslant \alpha_{2}^{\star} \Lambda \sup \mu_{\mathrm{P}}(y)=\alpha_{2}^{\star} \\
& y \in \underline{H}_{\alpha}^{\star}{ }_{1}^{\star}-\Delta \alpha \quad y \in \underline{H}_{\alpha}^{\star}
\end{aligned}
$$

Since $\left(\alpha_{1}^{\star}-\Delta \alpha\right)>\alpha_{2}^{\star}$ and $\left(\alpha_{1}^{\star}-\Delta \alpha\right) \Lambda \sup \mu_{\mathrm{P}}(\mathrm{y}) \leqslant \alpha_{2}^{\star}$

$$
y \in \underline{H}_{\alpha}^{\star}-\Delta \alpha
$$

in order to realize these two conditions, it is necessary that:

$$
\begin{aligned}
& \sup \mu_{\mathrm{P}}(y) \leqslant \alpha_{2}^{\star}=\sup _{\mathrm{y} \in \underline{H}_{-}^{\star}{ }_{1}-\Delta} \mu_{\mathrm{P}}(\mathrm{y}) \quad \text { (by assumption) } \\
& y \in \underline{H}_{\alpha}^{\star}
\end{aligned}
$$

Since $\mathrm{T}=\varnothing, \forall \mathrm{y} \in \underline{\mathrm{H}}_{\alpha_{1}^{\star}}, \mu_{\mathrm{P}}(\mathrm{y}) \neq 1$.

According to the continuity of $\mu_{\mathrm{P}}$, we can choose $\Delta \alpha$ such that $\forall y \in \underline{\mathrm{H}}_{\alpha-\Delta \alpha}, \mu_{\mathrm{P}}(\mathrm{y}) \neq 1$.

Since $\underline{H}_{\alpha-\Delta \alpha}$ is closed and since $\mu_{\mathrm{P}}(\mathrm{y})$ is strongly quasi-concave, the value $y_{1}$ which maximizes $\mu_{\mathrm{P}}(\mathrm{y})$ in $\underline{\mathrm{H}}_{\alpha_{1}}-\Delta \alpha^{\prime}$ belongs to the frontier of $\underline{H}_{\alpha}^{\star}-\Delta \alpha$, by virtue of Lemma 1 .

According to Lemma $2, \forall y \in \underline{H}_{\alpha} \Longrightarrow y \in \underline{H}_{\alpha_{1}}-\Delta \alpha$

By ${ }_{0}$ contraposition, we write:

$\mathrm{y}_{1} \notin \stackrel{\mathrm{H}}{\alpha}_{1}-\Delta \alpha \Longrightarrow \mathrm{y}_{1} \notin \mathrm{H}_{\alpha_{1}}$

follows that:

By virtue of the strong quasi-concavity of $\mu_{P}(y)$, it

$$
\begin{aligned}
& \sup _{\mu_{p}}(y)>\sup \mu_{p}(y) \\
& y \in \underline{H}_{\alpha_{1}^{\star}-\Delta \alpha} \quad y \in \underline{H}_{\alpha_{1}^{\star}}{ }_{1}
\end{aligned}
$$


which contradicts $\sup \mu_{\mathrm{P}}(y) \geqslant \sup \mu_{\mathrm{P}}(y)$

$$
y \in \underline{H}_{\alpha}^{\star} \quad y \in \underline{H}_{\alpha}^{\star}{ }_{1}^{\star}-\Delta \alpha
$$

(3.2.5.3.4.) In case 4 , let us assume that:

$$
\alpha_{1}^{\star} \geqslant \sup _{y \in \underline{H}_{\alpha_{2}} \mu_{2}(y)}=\sup _{\alpha_{\mathrm{P}}}(y)
$$

and it suffices to use the demonstration developed in (3.2.5.3.3.) to arrive at a contradiction.

$$
\text { [Q.E.D.] }
$$

3.2.6. At the equilibrium determined in this way, the fuzzy supply provides the producer with the greatest fuzzy utility of profit with respect to a given spatial price system and under the fuzzy technological constraint.

In the outputs demand space, the selling places of the products and the respective quantities sold there, are determined simultaneously. Similarly, in the inputs supply space, the places where the factors of production are purchased and the respective quantities acquired are obtained simultaneously. We recall that an equilibrium production is an element $\left(y_{i}^{r}\right)$ of the set $\underline{Y}$, with $i=1, \ldots, m$ and $r=1, \ldots, q$, where $i=1, \ldots, n$ designate the inputs, and $r=1, \ldots, p$ their supply points and where $i=n+1, \ldots, m$ stand for the outputs and $r=p+1, \ldots, q$ their demand points.

In general, the solution is not unique since the unicity conditions prove to be very restrictive and all these conditions can only be satisfied in a very particular case. 


\section{CONCLUSION}

4.1. Turning to the theory of fuzzy subsets makes it possible to determine the producer's spatial equilibrium in an imprecise context by enabling us to formalize, in a rigorous way, the economic calculation of the maximisation of the fuzzy utility of profit under a fuzzy technological constraint and with respect to a given spatial price system.

4.2. This analysis should not be contrasted with the classical theory of the producer, for the former contains the latter as a particular case: profit maximisation producing the maximal utility of profit under a rigid technological constraint.

4.3. This generalisation is not the only outcome of the application of the theory of fuzzy subsets. New results are also achieved. Equilibrium is no longer necessarily determined at a point or at a set of points belonging to a subset of the frontier of the set of all possible productions. Furthermore, the convexity assumption proves to be less restrictive on the production sets in this theory than in the classical theory. Indeed, on the one hand, in the theory of fuzzy subsets, the convexity is by definition a weakened convexity, and, on the other hand, in the framework of spatial analysis, the convexity hypothesis often seems non constraining, owing to the specific effect of transport prices.

4.4. This type of model requires developments. The dynamic aspect deserves to be integrated in terms of the maximisation of a fuzzy expected utility of profit under a fuzzy random constraint (imprecise reliability).

Final1y, the problem of the optimal location of the production unit in a fuzzy universe remains to be solved (the passage from the short to the long period). 


\section{BIBLIOGRAPHY}

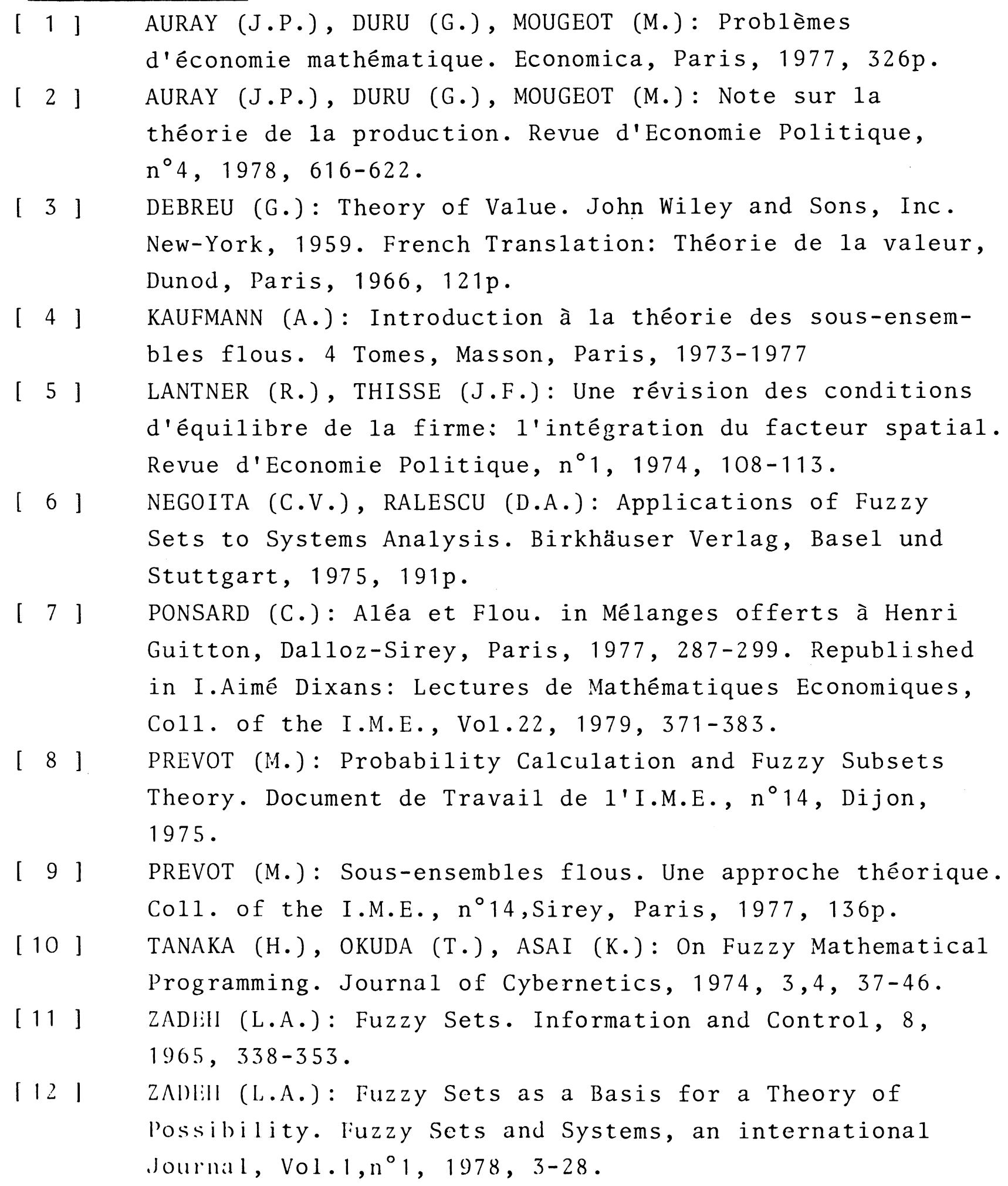

